\title{
Vegetation pattern formation of a water-biomass model*
}

\author{
Xiaoli Wang, Wendi Wang†, Guohong Zhang \\ Key Laboratory of Eco-environments in Three Gorges Reservoir Region, \\ School of Mathematics and Statistics, Southwest University, \\ Chongqing, 400715, P.R. China.
}

\begin{abstract}
In this paper, a mathematical model with diffusion and cross-diffusion is proposed to describe the interaction between the vegetation and the soil water. Based on the view of Turing pattern, we discuss the conditions of the diffusioninduced instability and the cross-diffusion-induced instability of a homogenous uniform steady state. We find that either a fast diffusion speed of water or a great hydraulic diffusivity due to the suction of roots may drive the instability of the homogenous steady state. Furthermore, we find that both the rain-fall rate and the infiltration feedback parameter can induce the transitions among the vegetation state, pattern formation and bare soil state. It is also found that the "terrain slope" may cause the instability of the homogenous steady state and drive the formation of periodic stripe pattern. Consequently, the diversity of dryland vegetation in reality can be explained as a result of pattern solutions of the model.
\end{abstract}

Keywords: Reaction-diffusion system; Cross-diffusion; Turing pattern; Pattern solution.

\section{Introduction}

In the past four decades, the ecological unbalance between the limited water resource and ecosystem engineers [14,15], such as animals, plants or microorganisms, has been

${ }^{*}$ This work is supported by grants from National Science Foundation of China $(11571284,11461023)$.

${ }^{\dagger}$ Corresponding Author. Email: wendi@swu.edu.cn

(C) 2016. This manuscript version is made available under the Elsevier user license http://www.elsevier.com/open-access/userlicense/1.0/ 
observed and the desertification has become more and more serious [10]. The desertification may be a slow and gradual process in which the vegetation is not homogenous but self-organized spatial patterns $[3,25,30]$. The vegetation spatial patterns exhibit repetitive or distinctive patches in space and time. Their forms can be a two-phased irregular mosaic consisting of a high-cover phase and a low-cover phase (bare land) [2] or many different regular types such as bands, labyrinth, spots, stripes, gaps, and rings $[16,17,23,24,30,31,35]$, which are observed in arid and semiarid areas.

How ecosystem engineers affect ecosystems and how the vegetation consists of patterns are the main frontiers in ecology [30] and have fascinated many ecologists $[14,15,40]$. Several mechanisms have been elucidated [40] to underly patterning of vegetation. Basically, these mechanisms include water scarcity, plant competition over water resources, redistribution of water by diffusion and runoff, and the positive feedback between water availability and plant [24, 28, 31, 33, 39]. Other factors, such as livestock overgrazing [29], climate variables [1, 6, 8], soil properties [38], rainfall interception [13, 32], and toxic compounds [4, 21, 22] may also drive the formation of patterns. Recently, mathematical modeling has been shown to be a powerful approach to understand mechanisms of pattern formations [10, 16, 18, 31, 30, 35, 39]. Based on the view of vegetation pattern formation as a symmetry-breaking phenomenon [5], the Klausmeier's model [16] generates the complex spatial patterns, often called a Turing pattern [37], from a relatively simple dynamical system.

To understand the mechanism for generation of vegetation patterns and their observed resilience, Shnerb et al. [35] propose the following mathematical model of one species (shrubs or trees) and one resource (water):

$$
\left\{\begin{array}{l}
\frac{\partial w}{\partial t}=D_{w} \Delta w+\mathbf{v} \cdot \nabla w+R-\lambda w b-w \\
\frac{\partial b}{\partial t}=w b-\mu b \\
w(x, 0)=w_{0}(x), b(x, 0)=b_{0}(x)
\end{array}\right.
$$

where $w$ is the ground water density and $b$ is the shrubs biomass density; parameters $D_{w}, R, \lambda, \mu$ are positive constants that denote the water diffusion coefficient, the rainfall rate, the water consumption rate in the presence of vegetation and the vegetation death rate, respectively; the term $\mathbf{v} \cdot \nabla w$ takes account of the downhill water loss in which $\mathbf{v}$ is a "terrain slope" vector. The initial states $w_{0}(x)$ and $b_{0}(x)$ are nonnegative 
functions of spatial variable $x$. The initial value problem of (1.1) is subject to a spatially periodic boundary condition over a planar spatial domain.

In [35], Shnerb et al. have shown that the final state of (1.1) is a uniform covering of all the plane by the amount of flora that corresponds to the stable fixed point. Note that there is no cross-diffusion in (1.1). Note also that the water infiltration at vegetation is important $[9,10]$. Following $[11,19,39]$, we modify the self-diffusion of water in $(1.1)$ to $D_{w} \Delta(w-\beta b)$, where $\beta>0$ represents the hydraulic diffusivity due to the suction of roots in the vadose zone. To capture the "infiltration feedback" between the plant and the ground water $[9,10]$, following [20] we assume that the vegetation death rate $\mu$ is monotonously decreasing in $b$ and is described by

$$
\mu(b)=\mu_{0}+\frac{\mu_{1}}{b+1}
$$

where $\mu_{0}, \mu_{1}$ are positive constants. Biologically, it can be explained as follows. The plant can loosen the soil locally which in turn increases the infiltration of the vegetation patch. Thus, the larger plant biomass density results in the higher infiltration, which decreases the death rate of shrubs due to the more soil water available. As a consequence, we consider the following model:

$$
\begin{cases}\frac{\partial w}{\partial t}=D_{w} \Delta(w-\beta b)+v \frac{\partial w}{\partial x_{1}}+R-\lambda w b-w, & x \in \Omega, t>0 \\ \frac{\partial b}{\partial t}=D_{b} \Delta b+w b-\mu(b) b, & x \in \Omega, t>0 \\ \frac{\partial w(x, t)}{\partial \nu}=\frac{\partial b(x, t)}{\partial \nu}=0, & x \in \partial \Omega, t>0 \\ w(x, 0)=w_{0}(x) \geq 0, b(x, 0)=b_{0}(x) \geq 0, & x \in \Omega .\end{cases}
$$

where all quantities are in nondimensional form; $\Omega$ is a bounded planar domain with a smooth boundary $\partial \Omega$; no-flux boundary condition is imposed on $\partial \Omega$ so that the ecosystem is closed to exterior environment; $\nu$ is the outward unit normal vector of the boundary $\partial \Omega ; x=\left(x_{1}, x_{2}\right)$ is the spatial variable; $\Delta=\frac{\partial}{\partial x_{1}^{2}}+\frac{\partial}{\partial x_{2}^{2}}$ is the Laplacian operator; the diffusion term $D_{b} \Delta b$ denotes the spread of plants both by clonal reproduction and by seed dispersal [26], where $D_{b}$ is a positive constant. Moreover, the surface runoff is modeled by the term $v \frac{\partial w}{\partial x_{1}}$, where $v$ is a constant downhill runoff flow velocity in the negative $x_{1}$-direction [16].

This paper is organized as follows. In Section 2, we analyze the diffusion-induced instability. In Section 3, we consider the cross-diffusion-induced instability. The effect of the ground surface is shown in Section 4. We end with discussions in Section 5. 


\section{Diffusion-induced Instability}

We start to consider the diffusion-induced instability, which means that the uniform steady state loses its stability due to the diffusion effect. When $\beta=0$ and $v=0$, we have the following initial boundary problem

$$
\begin{cases}\frac{\partial w}{\partial t}=D_{w} \Delta w+R-\lambda w b-w, & x \in \Omega, t>0, \\ \frac{\partial b}{\partial t}=D_{b} \Delta b+w b-\left(\mu_{0}+\frac{\mu_{1}}{b+1}\right) b, & x \in \Omega, t>0, \\ \frac{\partial w(x, t)}{\partial \nu}=\frac{\partial b(x, t)}{\partial \nu}=0, & x \in \partial \Omega, t>0, \\ w(x, 0)=w_{0}(x) \geq 0, b(x, 0)=b_{0}(x) \geq 0, & x \in \Omega .\end{cases}
$$

Here, we consider system (2.1) in the spatial domain $\Omega=(0, l \pi)$. Firstly, we present the following lemma about the global stability of the bare-soil steady state $\left(w_{0}, b_{0}\right)=(R, 0)$, which means the extinction of the plant.

Lemma 2.1. Let the parameters $D_{w}, D_{b}, R, \lambda, \mu_{0}, \mu_{1}$ be positive. Then the bare-soil steady state $\left(w_{0}, b_{0}\right)$ of $(2.1)$ is globally asymptotically stable if $R \leq \mu_{0}$.

Proof. Choose a Lyapunov function

$$
E(t):=\int_{\Omega}\left(w-w_{0}-w_{0} \ln \frac{w}{w_{0}}+\lambda b\right) d x .
$$

Its derivative along the solutions of $(2.1)$ is

$$
\begin{aligned}
E^{\prime}(t)= & \int_{\Omega}\left\{\left(1-\frac{w_{0}}{w}\right) w_{t}+\lambda b_{t}\right\} d x \\
= & \int_{\Omega}\left\{\left(1-\frac{w_{0}}{w}\right) D_{w} \Delta w+\lambda D_{b} \Delta b\right\} d x \\
& +\int_{\Omega}\left\{\left(1-\frac{w_{0}}{w}\right)(R-\lambda w b-w)+\lambda b\left(w-\mu_{0}-\frac{\mu_{1}}{1+b}\right)\right\} d x \\
= & -\int_{\Omega} \frac{w_{0} D_{w}}{w^{2}}|\nabla w|^{2} d x \\
& -\int_{\Omega}\left\{R\left(\frac{w}{w_{0}}+\frac{w_{0}}{w}-2\right)+\frac{\lambda b}{(1+b)}\left[\left(\mu_{0}-R\right) b+\left(\mu_{0}+\mu_{1}-R\right)\right]\right\} d x .
\end{aligned}
$$

Note that

$$
\frac{w}{w_{0}}+\frac{w_{0}}{w}-2 \geq 0
$$

and

$$
\left(\mu_{0}-R\right) b+\left(\mu_{0}+\mu_{1}-R\right)>0
$$


for all $b>0$ if $R \leq \mu_{0}$. It follows that $E^{\prime}(t) \leq 0$ and $E^{\prime}(t)=0$ if and only if $(w(x, t), b(x, t))=\left(w_{0}, b_{0}\right)$. Hence, all the solutions of system (2.1) approach the baresoil equilibrium $\left(w_{0}, b_{0}\right)=(R, 0)$ as $t \rightarrow \infty$ if $R \leq \mu_{0}$.

From Lemma 2.1, it suffices to consider the case of $R>\mu_{0}$ to establish the vegetation pattern formation of system (2.1). Set

$$
X:=\left\{(w, b) \in H^{2}[(0, l \pi)] \times H^{2}[(0, l \pi)]: w^{\prime}(0)=b^{\prime}(0)=w^{\prime}(l \pi)=b^{\prime}(l \pi)=0\right\} .
$$

The complexification of $X$ is

$$
X_{\mathbf{C}}:=X \oplus i X=\left\{x_{1}+i x_{2} \mid x_{1}, x_{2} \in X\right\}
$$

Let $\left(w^{+}, b^{+}\right)$be a stationary homogeneous solution of system (2.1). The linearized operator of system $(2.1)$ at $\left(w^{+}, b^{+}\right)$is

$$
L:=\left(\begin{array}{cc}
D_{w} \frac{\partial^{2}}{\partial x^{2}}+a_{11} & a_{12} \\
a_{21} & D_{b} \frac{\partial^{2}}{\partial x^{2}}+a_{22}
\end{array}\right),
$$

where

$$
\begin{array}{lrl}
a_{11}=-\lambda b^{+}-1<0, & a_{12}=-\frac{\lambda R}{\lambda b^{+}+1}<0, \\
a_{21}=b^{+}>0, & a_{22}=\frac{\mu_{1} b^{+}}{\left(b^{+}+1\right)^{2}}>0 .
\end{array}
$$

It is well known that the eigenvalue problem

$$
-\Phi^{\prime \prime}=\rho \Phi, \quad x \in(0, l \pi), \quad \Phi^{\prime}(0)=\Phi^{\prime}(l \pi)=0
$$

has eigenvalues $\rho_{k}=k^{2} / l^{2}, k=0,1,2, \ldots$, with corresponding eigenfunctions $\Phi_{k}(x)=$ $\cos \frac{k}{l} x$. Let

$$
\left(\begin{array}{l}
\phi \\
\psi
\end{array}\right)=\sum_{k=0}^{\infty}\left(\begin{array}{c}
\phi_{k} \\
\psi_{k}
\end{array}\right) \cos \frac{k}{l} x
$$

be an eigenfunction for $L$ with eigenvalue $\sigma$, i.e., $L(\phi, \psi)^{T}=\sigma(\phi, \psi)^{T}$. From [41], there exits $k \in \mathbf{N}_{0}$ such that $L_{k}\left(\phi_{k}, \psi_{k}\right)^{T}=\sigma\left(\phi_{k}, \psi_{k}\right)^{T}$, where $L_{k}$ is defined by

$$
L_{k}=L_{k}\left(R, \mu_{1}, r\right):=\left(\begin{array}{cc}
-\frac{D_{w} k^{2}}{l^{2}}+a_{11} & a_{12} \\
a_{21} & -\frac{r D_{w} k^{2}}{l^{2}}+a_{22}
\end{array}\right)
$$

with $r=D_{b} / D_{w}$. The characteristic equation of $L_{k}\left(R, \mu_{1}, r\right)$ in $\sigma$ is

$$
\sigma^{2}-\operatorname{Tr}_{k}\left(R, \mu_{1}, r\right) \sigma+\operatorname{Det}_{k}\left(R, \mu_{1}, r\right)=0, k=0,1,2, \ldots
$$


where

$$
\begin{aligned}
\operatorname{Tr}_{k}\left(R, \mu_{1}, r\right) & =-\frac{k^{2}}{l^{2}}(1+r) D_{w}+\operatorname{Tr}_{0}\left(R, \mu_{1}\right) \\
& =-\frac{k^{2}}{l^{2}}(1+r) D_{w}+a_{11}+a_{22}, \\
\operatorname{Det}_{k}\left(R, \mu_{1}, r\right) & =r D_{w}^{2} \frac{k^{4}}{l^{4}}-\left(r a_{11}+a_{22}\right) D_{w} \frac{k^{2}}{l^{2}}+\operatorname{Det}_{0}\left(R, \mu_{1}\right) \\
& =r D_{w}^{2} \frac{k^{4}}{l^{4}}-\left(r a_{11}+a_{22}\right) D_{w} \frac{k^{2}}{l^{2}}+a_{11} a_{22}-a_{12} a_{21} .
\end{aligned}
$$

To determine conditions for the instability of $\left(w^{+}, b^{+}\right)$with respect to $(2.1)$, we always assume that $\left(w^{+}, b^{+}\right)$is linearly stable with respect to the corresponding kinetic system, i.e.,

$$
\operatorname{Tr}_{0}\left(R, \mu_{1}\right)<0, \operatorname{Det}_{0}\left(R, \mu_{1}\right)>0
$$

It is easy to examine $\operatorname{Det}_{0}\left(R, \mu_{1}\right)>0$. Thus, let us assume

$$
\operatorname{Tr}_{0}\left(R, \mu_{1}\right)=a_{11}+a_{22}=\frac{b^{+} \mu_{1}}{\left(b^{+}+1\right)^{2}}-\left(\lambda b^{+}+1\right)<0 .
$$

Set

$$
r_{c}=\frac{\frac{D_{w}}{l^{2}} a_{22}+a_{11} a_{22}-2 a_{12} a_{21}-2 \sqrt{\left(\frac{D_{w}}{l^{2}} a_{22}-a_{12} a_{21}\right)\left(a_{11} a_{22}-a_{12} a_{21}\right)}}{\left(\frac{D_{w}}{l^{2}}-a_{11}\right)^{2}} .
$$

Then we have the following result of diffusion-induced instability:

Theorem 2.2. Assume that all of parameters $R, \lambda, \mu_{0}, \mu_{1}, l, D_{w}, D_{b}$ are positive and $r=D_{b} / D_{w}$. Suppose that $\beta=0, v=0$, and (2.9) is satisfied. If

$$
r \leq r_{c}
$$

then $\left(w^{+}, b^{+}\right)$is an unstable uniform state of the reaction-diffusion system (2.1). That is, the diffusion induces instability.

Proof. We aim to get a diffusive instability from the uniform steady state that is stable to linear homogeneous perturbations. By $[27,34]$ we need the following condition

$$
\operatorname{Det}_{k}(r)=r D_{w}^{2} \frac{k^{4}}{l^{4}}-\left(r a_{11}+a_{22}\right) D_{w} \frac{k^{2}}{l^{2}}+a_{11} a_{22}-a_{12} a_{21}<0
$$

for some positive integer $k$ to ensure that one of the eigenvalues of $L_{k}$ has a positive real part. Clearly, a necessary condition for that is

$$
r a_{11}+a_{22}>0
$$


Notice that $\operatorname{Det}_{k}(r)$ achieves its minimum

$$
\min _{k \in \mathbf{R}^{+}} \operatorname{Det}_{k}=a_{11} a_{22}-a_{12} a_{21}-\frac{\left(r a_{11}+a_{22}\right)^{2}}{4 r}
$$

at the critical value $k_{c}$ which satisfies

$$
k_{c}^{2}=\frac{\left(r a_{11}+a_{22}\right) l^{2}}{2 r D_{w}} .
$$

Then a necessary condition for the instability of $\left(w^{+}, b^{+}\right)$with respect to $(2.1)$ is

$$
\min _{k \in \mathbf{R}^{+}} \operatorname{Det}_{k}\left(R, \mu_{1}, r\right)<0, \quad \text { and } \quad k_{c}^{2}>0
$$

which is equivalent to

$$
r a_{11}+a_{22}>2 \sqrt{a_{11} a_{22}-a_{12} a_{21}} \sqrt{r}
$$

i.e.,

$$
0<r<r_{*}:=\frac{a_{11} a_{22}-2 a_{12} a_{21}-2 \sqrt{-a_{12} a_{21}\left(a_{11} a_{22}-a_{12} a_{21}\right)}}{a_{11}^{2}} .
$$

However, for a finite domain (2.16) is necessary but not sufficient for instability [34]. Let $k_{1}^{2}$ and $k_{2}^{2}$ be the two positive roots of $\operatorname{Det}_{k}(r)=0$. Then

$$
\begin{aligned}
& k_{1}^{2}=\frac{\left(\left(r a_{11}+a_{22}\right) D_{w}-\sqrt{\left(r a_{11}+a_{22}\right)^{2} D_{w}^{2}-4 r D_{w}^{2}\left(a_{11} a_{22}-a_{12} a_{21}\right)}\right) l^{2}}{2 r D_{w}^{2}}, \\
& k_{2}^{2}=\frac{\left(\left(r a_{11}+a_{22}\right) D_{w}+\sqrt{\left(r a_{11}+a_{22}\right)^{2} D_{w}^{2}-4 r D_{w}^{2}\left(a_{11} a_{22}-a_{12} a_{21}\right)}\right) l^{2}}{2 r D_{w}^{2}},
\end{aligned}
$$

and $0<k_{1}^{2}<k_{c}^{2}<k_{2}^{2}$. Thus, in order to obtain the instability of $\left(w^{+}, b^{+}\right)$, we must have $0<k_{1}^{2}<k^{2}<k_{2}^{2}$ for some positive integer $k$, and a necessary condition is $k_{2}-k_{1} \geq 1$, which results in

$$
\frac{l^{2}}{r D_{w}}\left(r a_{11}+a_{22}-2 \sqrt{a_{11} a_{22}-a_{12} a_{21}} \sqrt{r}\right) \geq 1 .
$$

Set

$$
g(\sqrt{r}):=\left(\frac{D_{w}}{l^{2}}-a_{11}\right) r+2 \sqrt{a_{11} a_{22}-a_{12} a_{21}} \sqrt{r}-a_{22}
$$

It follows from (2.17) and (2.20) that $\left(w^{+}, b^{+}\right)$is unstable with respect to (2.1) if $g(\sqrt{r}) \leq 0$. Note that $a_{11}<0$ and $a_{22}>0$. Then $g(\sqrt{r})=0$ has a unique positive root $r_{c}$, which is defined as in (2.10). Then $g(\sqrt{r}) \leq 0$ when $0<r \leq r_{c}$. This completes the proof. 


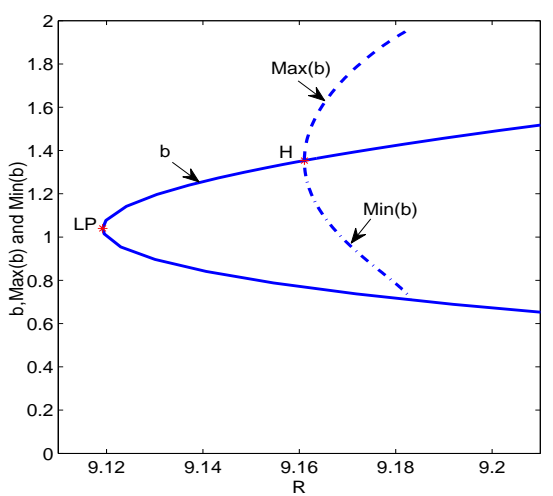

Figure 1: Bifurcation diagram of the corresponding kinetic system of (2.1) when $\lambda=$ $0.2, \mu_{0}=5, \mu_{1}=5.2$. The solid curve, the dot-dashed curve and the dashed curve represent the biomass $b$, the minimum value and the maximum value of $b$ of the limit cycles vs. the rain-fall rate $R$.

Example 2.3. For illustration purpose, we fix parameters as $\lambda=0.2, \mu_{0}=5, \mu_{1}=$ 5.2. Then numerical simulations from MatCont [7] for the Bifurcation diagram of the corresponding kinetic model of system (2.1) is shown in Fig. 1. There are three critical values, denoted by $R_{0}, R_{1}$ and $R_{3}$, where $R_{0}=11.2$ (a backward transcritical bifurcation point), $R_{1}=9.119216$ (a saddle-node bifurcation point), and $R_{3}=9.161059$ (a subcritical Hopf bifurcation point). The corresponding kinetic system of (2.1) has a bare-soil equilibrium $(R, 0)$ for all parameters, has a unique positive equilibrium $\left(w^{+}, b^{+}\right)=\left(R /\left(\lambda b^{+}+1\right), b^{+}\right)$when $R=R_{1}$ or $R \geq R_{0}$, has two positive equilibria $\left(w^{ \pm}, b^{ \pm}\right)=\left(R /\left(\lambda b^{ \pm}+1\right), b^{ \pm}\right)$when $R_{1}<R<R_{0}$, and has no positive equilibrium when $R<R_{1}$. The bare-soil equilibrium $(R, 0)$ is stable when $R<R_{0}$, and unstable when $R>R_{0}$. The positive equilibrium $\left(w^{-}, b^{-}\right)$is always a saddle. The equilibrium $\left(w^{+}, b^{+}\right)$is unstable when $R_{1}<R<R_{3}$, and stable when $R>R_{3}$. If we choose $R=$ $14, D_{w}=10$ and $l=3$, then the positive equilibrium $\left(w^{+}, b^{+}\right)$is locally asymptotically stable for the corresponding kinetic model of (2.1). By (2.9) and (2.18), we have $r_{c}=0.0092$ and $r_{*}=0.0095$. According to Theorem 2.2 there exists an unbounded region $\left\{\left(D_{w}, D_{b}\right): D_{w}>0, D_{b}>0, D_{b} \leq 0.0092 D_{w}\right\}$ in which Turing instability occurs. There exists also an unbounded region $\left\{\left(D_{w}, D_{b}\right): D_{w}>0, D_{b}>0, D_{b} \geq 0.0095 D_{w}\right\}$ in which the stable equilibrium $\left(w^{+}, b^{+}\right)$remains stable. The linear growth factor curves $\sigma=\sigma(k)$ for perturbations with wavenumbers $k$ around the homogenous vegetation state when the ratio value $r$ below, at and beyond the instability are shown in Fig. 


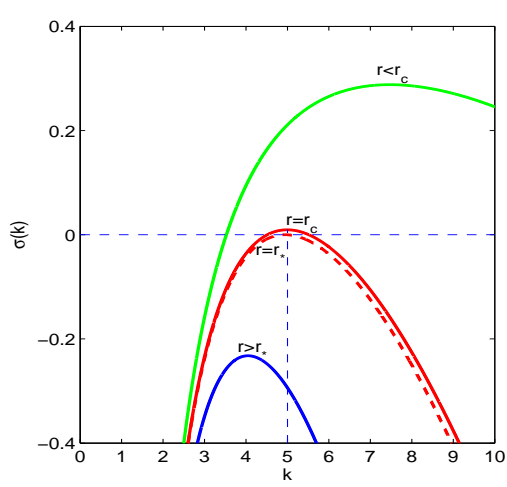

(a)

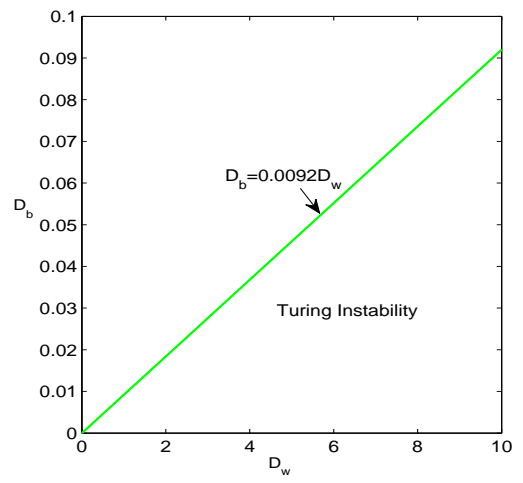

(b)

Figure 2: (a): The linear growth factor curves $\sigma=\sigma(k)$ for perturbations with wavenumbers $k$ around the homogenous vegetation state when the ratio value $r$ below, at and beyond the instability. (b): Turing instability only occurs below the green curve. Parameters: $R=14, \lambda=0.2, \mu_{0}=5, \mu_{1}=5.2, l=3, D_{w}=10$ in (a), (b) and $r=0.002,0.0092,0.0095,0.02$, respectively, in (a).

2 (a) where the corresponding wavenumber of unstable mode is $k=5$ when $r=r_{c}$. Note that $\sigma(5)<0$ when $r=r_{*}$. This implies that $\sigma(k)<0$ for all $k \geq 0$ when $r=r_{*}$. Then the positive equilibrium $\left(w^{+}, b^{+}\right)$remains stable in the narrow region $\left\{\left(D_{w}, D_{b}\right): D_{w}>0, D_{b}>0,0.0092 D_{w} \leq D_{b} \leq 0.0095 D_{w}\right\}$. The parameter space for the instability of $\left(w^{+}, b^{+}\right)$of $(2.1)$ is shown as in Fig. $2(\mathrm{~b})$ where $\left(w^{+}, b^{+}\right)$is unstable with respect to $(2.1)$ in the region $\left\{\left(D_{w}, D_{b}\right): D_{w}>0, D_{b}>0, D_{b} \leq 0.0092 D_{w}\right\}$. Choosing $r=0.002<r_{c}$, the pattern solutions of model (2.1) are shown in Fig. 3 where the higher water density area results in the higher density of biomass.

Based on Theorem 2.2, we now consider the effect of rain-fall rate $R$ and infiltration feedback parameter $\mu_{1}$ on the pattern formation. For the fixed $\mu_{1}$, it follows from Theorem 2.2 that system (2.1) admits pattern formation when $r \leq r_{c}(R)$, where

$$
r_{c}(R)=\frac{\frac{D_{w}}{l^{2}} a_{22}+a_{11} a_{22}-2 a_{12} a_{21}-2 \sqrt{\left(\frac{D_{w}}{l^{2}} a_{22}-a_{12} a_{21}\right)\left(a_{11} a_{22}-a_{12} a_{21}\right)}}{\left(\frac{D_{w}}{l^{2}}-a_{11}\right)^{2}},
$$

and no pattern formation when $r \geq r_{*}(R)$, where

$$
r_{*}(R):=\frac{a_{11} a_{22}-2 a_{12} a_{21}-2 \sqrt{-a_{12} a_{21}\left(a_{11} a_{22}-a_{12} a_{21}\right)}}{\left(-a_{11}\right)^{2}} .
$$




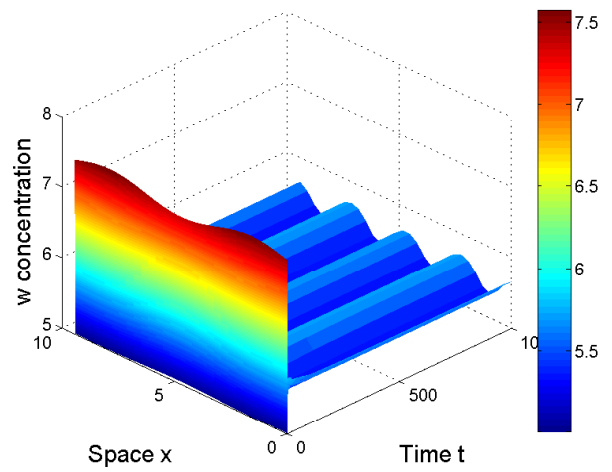

(a)

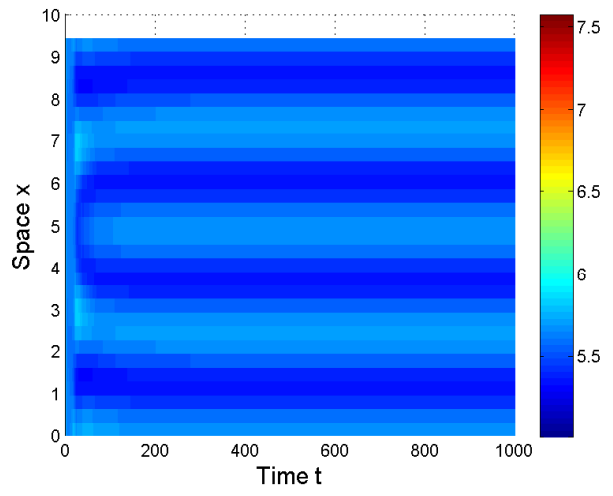

(c)

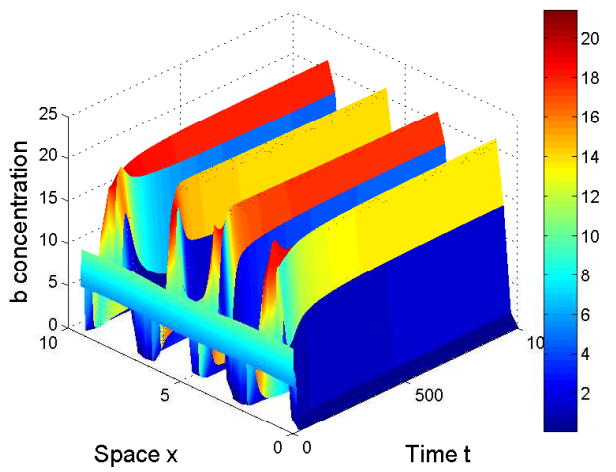

(b)

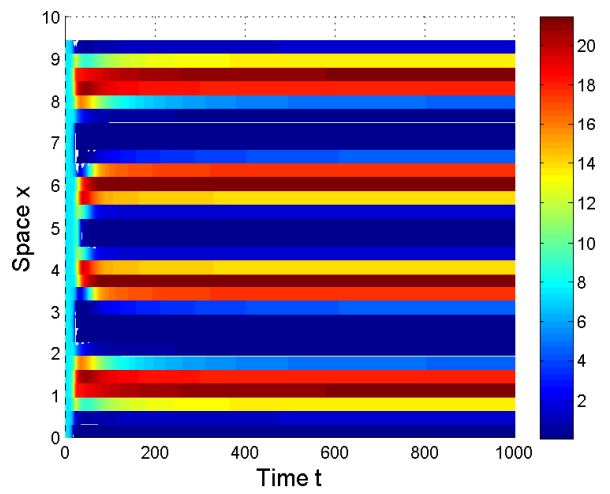

(d)

Figure 3: Pattern solutions which are induced by diffusion in model (2.1). Panel (a) and panel (b) show a 3D view of the concentration of biomass $w$ and $b$, respectively. Panel (c) and panel (d) show a 2D view of the concentration of biomass $w$ and $b$, respectively. Parameters: $R=14, \lambda=0.2, D_{w}=10, D_{b}=0.02, \mu_{0}=5, \mu_{1}=5.2, l=3$. 
Note that at the bare-soil equilibrium $(R, 0)$, we have

$$
\begin{aligned}
\operatorname{Tr}_{k}(R, r) & =-\frac{k^{2}}{l^{2}}(1+r) D_{w}+R-\left(\mu_{0}+\mu_{1}+1\right), \\
\operatorname{Det}_{k}(R, r) & =r D_{w}^{2} \frac{k^{4}}{l^{4}}+\left[r+\left(\mu_{0}+\mu_{1}\right)-R\right] D_{w} \frac{k^{2}}{l^{2}}+\left(\mu_{0}+\mu_{1}\right)-R .
\end{aligned}
$$

Clearly, if $R \leq \mu_{0}+\mu_{1}$, we have $\operatorname{Tr}_{k}(R, r)<0$ and $\operatorname{Det}_{k}(R, r)>0$ for all $k \geq 0$. Hence, the bare-soil equilibrium is local stable when $R \leq \mu_{0}+\mu_{1}$. We show in the following example that pattern formation may occur when $R>\mu_{0}+\mu_{1}$.

Example 2.4. Let us fix $\lambda=1.2, \mu_{0}=0.1, \mu_{1}=0.3$. It is easy to show that the corresponding kinetic system of (2.1) has no positive equilibrium when $R \leq \mu_{0}+\mu_{1}$ and has one unique positive equilibrium $\left(w^{+}, b^{+}\right)$which is locally asymptotically stable when $R>\mu_{0}+\mu_{1}$. If $D_{w}=10$ and $l=3$, by numerical computations we obtain the effect of the rain-fall rate on pattern formation which is shown in Fig. 4. Note that the vertical line $R=\mu_{0}+\mu_{1}$ separates the $R-r$ plane into two states where biomass is able to exist or not: the bare soil equilibrium is locally stable when $R \leq \mu_{0}+\mu_{1}$, and pattern formation or vegetation exists when $R>\mu_{0}+\mu_{1}$. More precisely, pattern formation occurs below the solid curve $r=r_{c}(R)$ and does not emerges above the dashed curve $r=r_{*}(R)$; In the region $\left\{(R, r): r_{c}(R)<r<r_{*}(R)\right\}$ the instability of $\left(w^{+}, b^{+}\right)$can be verified by numerical simulations. Therefore, these boundaries divide the parameter plane into three states: bare-soil, pattern formation, vegetation. Furthermore, for a larger $r$ (dashed line $\mathrm{A}$ ), as the rain-fall rate $R$ decreases, the transition is from the vegetation state to the bare soil state; For a smaller $r$ (dashed line B), as the rainfall rate $R$ decreases, there are multiple transitions among the vegetation, pattern formation and the bare soil state.

We now consider the effect of the infiltration feedback parameter $\mu_{1}$. For the fixed $R$, it follows from Theorem 2.2 that system (2.1) admits pattern formation when $r \leq$ $r_{c}\left(\mu_{1}\right)$, where

$$
r_{c}\left(\mu_{1}\right)=\frac{\frac{D_{w}}{l^{2}} a_{22}+a_{11} a_{22}-2 a_{12} a_{21}-2 \sqrt{\left(\frac{D_{w}}{l^{2}} a_{22}-a_{12} a_{21}\right)\left(a_{11} a_{22}-a_{12} a_{21}\right)}}{\left(\frac{D_{w}}{l^{2}}-a_{11}\right)^{2}},
$$

and no pattern formation when $r \geq r_{*}\left(\mu_{1}\right)$, where

$$
r_{*}\left(\mu_{1}\right):=\frac{a_{11} a_{22}-2 a_{12} a_{21}-2 \sqrt{-a_{12} a_{21}\left(a_{11} a_{22}-a_{12} a_{21}\right)}}{\left(-a_{11}\right)^{2}} .
$$




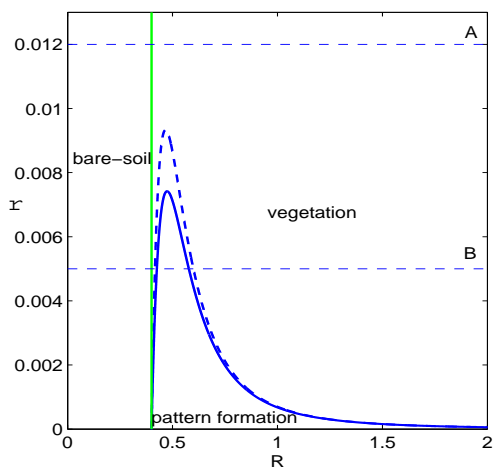

Figure 4: The graph shows effects of rain-fall rate on the transition of three states: bare-soil, pattern formation, vegetation, where $\lambda=1.2, \mu_{0}=0.1, D_{w}=10, l=3$ and $\mu_{1}=0.3$.

By similar discussions to $(2.24)$ at the bare-soil equilibrium $(R, 0)$ we see that $\operatorname{Tr}_{k}\left(\mu_{1}, r\right)<$ 0 and $\operatorname{Det}_{k}\left(\mu_{1}, r\right)>0$ for all $k \geq 0$ if $\mu_{1}>R-\mu_{0}$. Hence, the bare-soil equilibrium is locally stable when $\mu_{1}>R-\mu_{0}$. The following example indicates that pattern

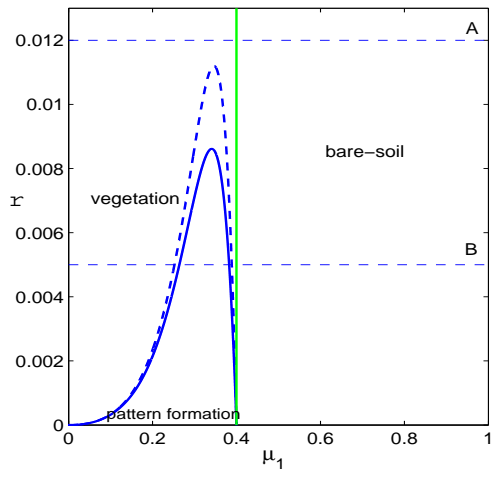

Figure 5: Effects of the infiltration feedback on the transition of three states: bare-soil, pattern formation, vegetation for $\lambda=1.2, \mu_{0}=0.1, D_{w}=10, l=3$ and $R=0.5$.

formation could occur for $\mu_{1}<R-\mu_{0}$.

Example 2.5. Fix the parameters by setting $\lambda=1.2, \mu_{0}=0.1, D_{w}=10, R=0.5$ and $l=3$. By numerical calculations we obtain Fig. 5 that presents the states where biomass is able to exist or not. Specifically, when $\mu_{1}>R-\mu_{0}$, the bare soil equilibrium is locally stable; when $\mu_{1}<R-\mu_{0}$, vegetation or pattern formation exists. In fact, pattern formation occurs below the solid curve $r=r_{c}\left(\mu_{1}\right)$, and does not occur above the dashed curve $r=r_{*}\left(\mu_{1}\right)$. Moreover, $\left(w^{+}, b^{+}\right)$may be unstable in the region $\left\{\left(\mu_{1}, r\right)\right.$ : 
$\left.r_{c}\left(\mu_{1}\right)<r<r_{*}\left(\mu_{1}\right)\right\}$. Furthermore, for a larger $r$ (dashed line A), as $\mu_{1}$ increases, the transition is from the vegetation state to the bare soil state, while for a medium $r$ (dashed line $\mathrm{B}$ ), as $\mu_{1}$ increases, there are multiple transitions among the vegetation, pattern formation and the bare soil state.

\section{Cross-diffusion-induced instability}

In this section, we consider the cross-diffusion-induced instability, which means that the uniform steady state loses its stability due to the cross- diffusion effect. When $\beta>0$ and $v=0$, the system reads

$$
\begin{cases}\frac{\partial w}{\partial t}=D_{w} \Delta(w-\beta b)+R-\lambda w b-w, & x \in \Omega, t>0, \\ \frac{\partial b}{\partial t}=D_{b} \Delta b+w b-\left(\mu_{0}+\frac{\mu_{1}}{b+1}\right) b, & x \in \Omega, t>0, \\ \frac{\partial w(x, t)}{\partial \nu}=\frac{\partial b(x, t)}{\partial \nu}=0, & x \in \partial \Omega, t>0, \\ w(x, 0)=w_{0}(x) \geq 0, b(x, 0)=b_{0}(x) \geq 0, & x \in \Omega .\end{cases}
$$

We still consider system (3.1) in the spatial domain $\Omega=(0, l \pi)$. Similar to the last section, the linearized operator $L$, with the domain $D(L)=X_{\mathbf{C}}$, of system (3.1) at $\left(w^{+}, b^{+}\right)$is

$$
L(\beta):=\left(\begin{array}{cc}
D_{w} \frac{\partial^{2}}{\partial x^{2}}+a_{11} & -\beta D_{w} \frac{\partial^{2}}{\partial x^{2}}+a_{12} \\
a_{21} & D_{b} \frac{\partial^{2}}{\partial x^{2}}+a_{22}
\end{array}\right),
$$

where $a_{11}, a_{12}, a_{21}, a_{22}$ are defined in (2.4). Let

$$
\left(\begin{array}{l}
\phi \\
\psi
\end{array}\right)=\sum_{k=0}^{\infty}\left(\begin{array}{l}
\phi_{k} \\
\psi_{k}
\end{array}\right) \cos \frac{k}{l} x
$$

be an eigenfunction for $L$ with eigenvalue $\sigma$, i.e., $L(\phi, \psi)^{T}=\sigma(\phi, \psi)^{T}$. Then there exists $k \in \mathbf{N}_{0}$ such that $L_{k}\left(\phi_{k}, \psi_{k}\right)^{T}=\sigma\left(\phi_{k}, \psi_{k}\right)^{T}$, where $L_{k}$ is defined by

$$
L_{k}=L_{k}(\beta, r):=\left(\begin{array}{cc}
-\frac{D_{w} k^{2}}{l^{2}}+a_{11} & -\frac{\beta D_{w} k^{2}}{l^{2}}+a_{12} \\
a_{21} & -\frac{r D_{w} k^{2}}{l^{2}}+a_{22}
\end{array}\right)
$$

in which the parameters except for $\beta$ and $r$ are fixed. The corresponding characteristic equation of $L_{k}(\beta)$ in $\sigma$ is

$$
\sigma^{2}-\operatorname{Tr}_{k}(\beta, r) \sigma+\operatorname{Det}_{k}(\beta, r)=0, \quad k=0,1,2, \ldots
$$


where

$$
\begin{aligned}
\operatorname{Tr}_{k}(\beta, r) & =-\frac{k^{2}}{l^{2}}(1+r) D_{w}+a_{11}+a_{22}:=\operatorname{Tr}_{k} \\
\operatorname{Det}_{k}(\beta, r) & =r D_{w}^{2} \frac{k^{4}}{l^{4}}-\left(r a_{11}+a_{22}+\beta a_{21}\right) D_{w} \frac{k^{2}}{l^{2}}+a_{11} a_{22}-a_{12} a_{21} .
\end{aligned}
$$

Set

$$
\operatorname{Det}_{k}=\operatorname{Det}_{k}(\beta, r)+\beta a_{21} D_{w} \frac{k^{2}}{l^{2}} .
$$

If $\operatorname{Det}_{k}<0$, then $\operatorname{Det}_{k}(\beta, r)<0$ is always true for $\beta>0, a_{21}=b^{+}>0$, and $D_{w}>0$. Hence, the cross-diffusion coefficient $\beta$ promotes the linear instability of the system $(3.1)$.

In the following, we assume that $\operatorname{Tr}_{k}<0$ and $\operatorname{Det}_{k}>0$, i.e., the uniform state $\left(w^{+}, b^{+}\right)$of $(3.1)$ is linearly stable, and discuss the cross-diffusion-induced instability. Since $\operatorname{Tr}_{k}<0$, in order to get a cross-diffusive instability from the uniform steady state that is stable to linear homogeneous perturbations, the following conditions are required $[27,34]$

$$
\operatorname{Det}_{k}(\beta, r)<0
$$

for some integer $k$ to ensure that one of the roots in (3.5) has a positive real part. A necessary condition for this is

$$
r a_{11}+a_{22}+\beta a_{21}>0
$$

Otherwise, $\operatorname{Det}_{k}(\beta, r)>0$ for all $k>0$. Notice that $\operatorname{Det}_{k}(\beta, r)$ achieves its minimum

$$
\min _{k \in \mathbf{R}^{+}} \operatorname{Det}_{k}(\beta, r)=a_{11} a_{22}-a_{12} a_{21}-\frac{\left(r a_{11}+a_{22}+\beta a_{21}\right)^{2}}{4 r}
$$

at the critical value $k_{c \beta}$ which satisfies

$$
k_{c \beta}^{2}=\frac{\left(r a_{11}+a_{22}+\beta a_{21}\right) l^{2}}{2 r} .
$$

Then a necessary condition for the cross-diffusion-induced instability is

$$
\min _{k \in \mathbf{R}^{+}} \operatorname{Det}_{k}(\beta, r)<0, \quad \text { and } \quad k_{c \beta}^{2}>0
$$

which is equivalent to

$$
r a_{11}+a_{22}+\beta a_{21}>2 \sqrt{a_{11} a_{22}-a_{12} a_{21}} \sqrt{r},
$$


i.e.,

$$
\beta>\beta_{*}(\sqrt{r}):=\frac{1}{a_{21}}\left(\left(-a_{11}\right) r+2 \sqrt{a_{11} a_{22}-a_{12} a_{21}} \sqrt{r}-a_{22}\right) .
$$

Similar to the proof of Theorem 2.2, we let

$$
\frac{l^{2}}{r D_{w}}\left(r a_{11}+a_{22}+\beta a_{21}-2 \sqrt{a_{11} a_{22}-a_{12} a_{21}} \sqrt{r}\right) \geq 1
$$

to ensure the cross-diffusion-induced instability. It follows from (3.11) and (3.12) that

$$
\beta \geq \beta_{c}(\sqrt{r}),
$$

where

$$
\beta_{c}(\sqrt{r}):=\frac{1}{a_{21}}\left(\left(\frac{D_{w}}{l^{2}}-a_{11}\right) r+2 \sqrt{a_{11} a_{22}-a_{12} a_{21}} \sqrt{r}-a_{22}\right) .
$$

Consequently, the following results can be stated.

Theorem 3.1. Assume that the parameters $R, \lambda, \mu_{0}, \mu_{1}$ are all positive. Suppose that $\beta>0, \mathbf{v}=0, D_{w}>0, D_{b}>0, \operatorname{Tr}_{k}<0$, and $\operatorname{Det}_{k}>0$. If

$$
\beta \geq \beta_{c}(\sqrt{r})
$$

then $\left(w^{+}, b^{+}\right)$is an unstable uniform state with respect to the cross-diffusion system (3.1), but is a stable equilibrium solution with respect to the reaction-diffusion system (2.1). That is, the cross-diffusion induces instability.

Remark 3.2. Note that $a_{11}<0, a_{22}>0$ and $a_{21}>0$. Then it is easy to show that $\beta_{c}(\sqrt{r})=0$ has one unique positive root $r_{c}$ which is shown in $(2.21)$.

Example 3.3. Let us fix $R=14, \lambda=0.2,\left(\mu_{0}, \mu_{1}\right)=(5,5.2), D_{w}=10, r=0.02$. By Theorem 2.1, the positive equilibrium $\left(w^{+}, b^{+}\right)$of system (2.2) is stable. By (3.12) and (3.15), we have

$$
\beta_{c}(\sqrt{r})=0.4827 r+0.7103 \sqrt{r}-0.0725,
$$

and

$$
\beta_{*}(\sqrt{r})=0.3339 r+0.7103 \sqrt{r}-0.0725 .
$$

According to (3.17) and (3.18), if $r=0.02$ the critical values are $\beta_{c}(\sqrt{0.02})=0.0376$ and $\beta_{*}(\sqrt{0.02})=0.0346$. The linear growth factor curves $\sigma=\sigma(k)$ for perturbations 


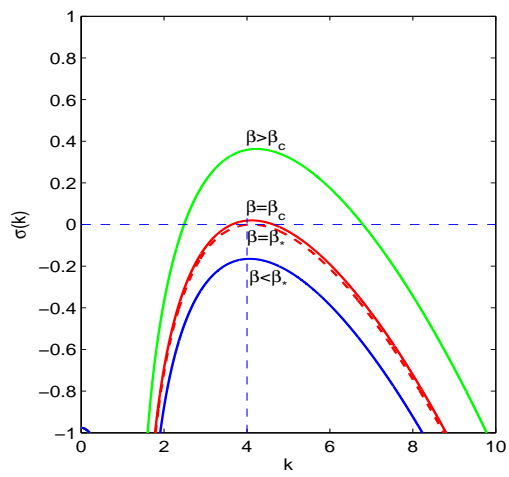

(a)

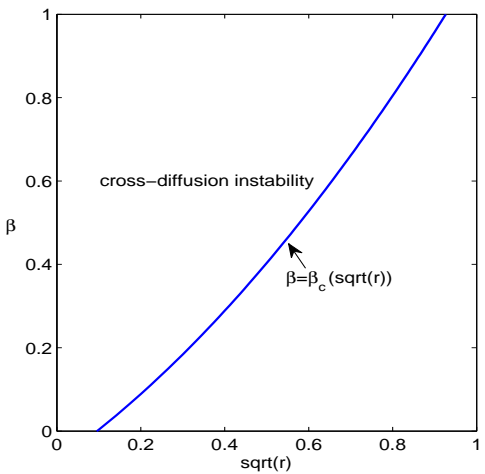

(b)

Figure 6: (a): The linear growth factor curves $\sigma=\sigma(k)$ for perturbations with wavenumbers $k$ around the homogenous vegetation state when the diffusion coefficient $\beta$ below, at and beyond the instability. (b): The instability threshold $\beta=\beta_{c}(\sqrt{r})$ in the plane spanned by the parameters $\beta$ and $\sqrt{r}$. Parameters: $R=14, \lambda=0.2, \mu_{0}=$ $5, \mu_{1}=5.2, D_{w}=10, l=3$ and in (a) $\beta=0.01,0.0376,0.0346,0.09$, respectively.

with wavenumbers $k$ around the homogenous vegetation state when the diffusion coefficient $\beta$ below, at and beyond instability are shown in Fig. 6 (a) where the wavenumber of unstable mode is $k=4$ when $\beta=\beta_{c}$. According to Theorem 3.1, the positive equilibrium $\left(w^{+}, b^{+}\right)$is stable in the region $\left\{(\sqrt{r}, \beta): \beta \leq \beta_{*}(\sqrt{r})\right\}$ and is unstable in the region $\left\{(\sqrt{r}, \beta): \beta \geq \beta_{c}(\sqrt{r})\right\}$. The fact that $\sigma(4)<0$ when $\beta=\beta_{*}$ implies that $\sigma(k)<0$ for all $k \geq 0$ when $\beta=\beta_{*}$. Then the positive equilibrium $\left(w^{+}, b^{+}\right)$is stable in the subregion $\left\{(\sqrt{r}, \beta): \beta_{*}(\sqrt{r})<\beta<\beta_{c}(\sqrt{r})\right\}$. The parameter space for cross-diffusion instability is shown in Fig. 6 (b) which indicates that cross-diffusion instability occurs only in the region $\left\{(\sqrt{r}, \beta): \beta \geq \beta_{c}(\sqrt{r})\right\}$. Choose $\beta=0.09>\beta_{c}$. Then according to Theorem 3.1, the cross-diffusion-induced instability occurs and the pattern solutions of model (3.1) are shown in Fig. 7.

From Theorem 3.1, function $\beta_{c}(\sqrt{r})$ is increasing with $\sqrt{r}$. Thus, the range of parameter $r$ for pattern formation is larger for a higher level of $\beta$. Biologically, parameter $\beta$ represents the ability of absorption of water due to the biomass and captures the "root-augmentation feedback" [25]. Thus, the larger $\beta$ means the stronger the rootaugmentation feedback. Note that the root system absorbs more water and thus slows down the diffusion speed of water around the localized spot as plant grows. It is natural that pattern solutions exist for a lager parameter range when the root-augmentation 


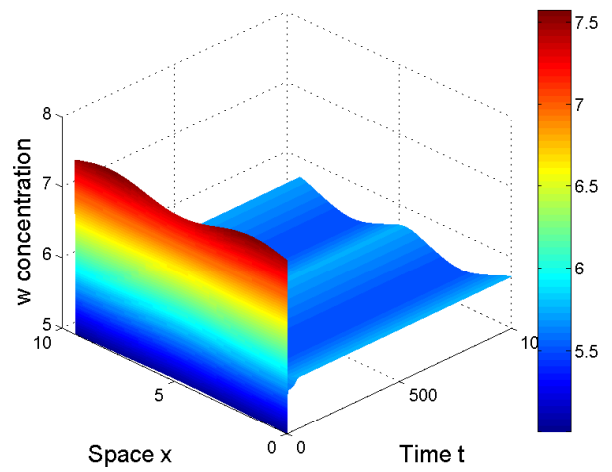

(a)

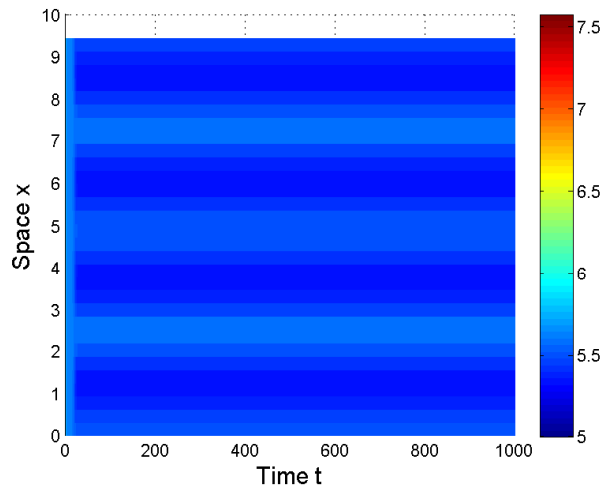

(c)

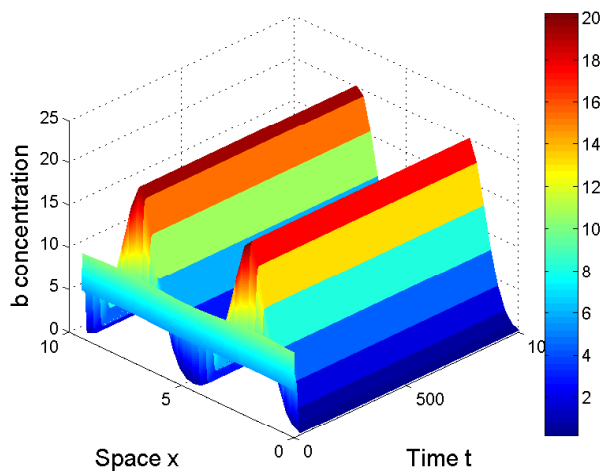

(b)

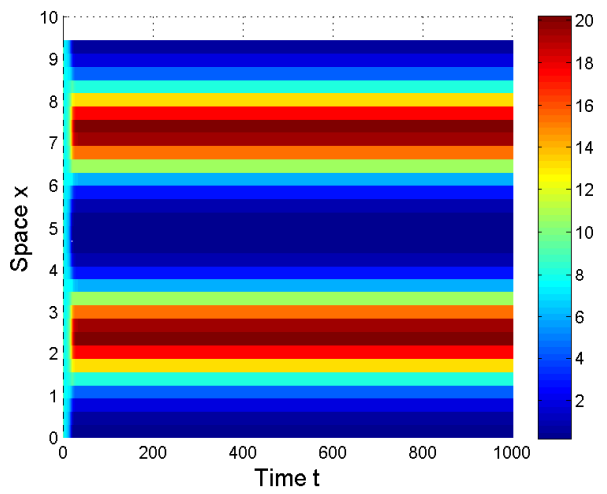

(d)

Figure 7: Pattern solutions which are induced by cross-diffusion of model (3.1). Panel (a) and panel (b) show a 3D view of the concentrations of biomass $w$ and $b$, respectively. Panel (c) and (d) show a 2D view of the concentrations of biomass $w$ and $b$, respectively. Parameters: $R=14, \lambda=0.2, \mu_{0}=5, \mu_{1}=5.2, D_{w}=10, D_{b}=0.2, l=3, \beta=0.09$. 
feedback is stronger.

\section{Effect of ground surface}

In this section, we consider the effect of ground surface on pattern formation in system (1.3). Let us assume $v \neq 0$ and $\beta=0$, which leads to the following initial boundary problem

$$
\begin{cases}\frac{\partial w}{\partial t}=D_{w} \Delta w+v \frac{\partial w}{\partial x_{1}}+R-\lambda w b-w, & x \in \Omega, t>0, \\ \frac{\partial b}{\partial t}=D_{b} \Delta b+w b-\left(\mu_{0}+\frac{\mu_{1}}{b+1}\right) b, & x \in \Omega, t>0, \\ \frac{\partial w(x, t)}{\partial \nu}=\frac{\partial b(x, t)}{\partial \nu}=0, & x \in \partial \Omega, t>0, \\ w(x, 0)=w_{0}(x) \geq 0, b(x, 0)=b_{0}(x) \geq 0, & x \in \Omega .\end{cases}
$$

Linearizing the reaction-diffusion system (4.1) about the stationary homogeneous solution $\left(w^{+}, b^{+}\right)$gives

$$
\left\{\begin{array}{l}
\frac{\partial \phi}{\partial t}=D_{w} \Delta \phi+v \frac{\partial w}{\partial x_{1}}+a_{11} \phi+a_{12} \psi \\
\frac{\partial \psi}{\partial t}=D_{b} \Delta \psi+a_{21} \phi+a_{22} \psi
\end{array}\right.
$$

We consider a perturbed solution of (4.2) in the form

$$
\Psi(t, x)=(\phi(t, x), \psi(t, x))^{T}=\left(\phi_{0}, \psi_{0}\right)^{T} \exp \left(\sigma_{\mathbf{k}} t+i \mathbf{k} \cdot \mathbf{r}\right)
$$

where $\sigma_{\mathbf{k}} \in \mathbf{R}$ is the linear growth factor, $\mathbf{k}=\left(k_{1}, k_{2}\right)$ is the wavevector, $\mathbf{r}$ is the directional vector. Substituting it into the system (4.2), we see that the nontrivial solution of this form exists provided that

$$
\begin{aligned}
\sigma_{\mathbf{k}}^{2} & +\left(-a_{11}-a_{22}+\left(D_{w}+D_{b}\right)\left(k_{1}^{2}+k_{2}^{2}\right)-i k_{1} v\right) \sigma_{\mathbf{k}} \\
& +\left(D_{w}\left(k_{1}^{2}+k_{2}^{2}\right)-a_{11}-i k_{1} v\right)\left(D_{b}\left(k_{1}^{2}+k_{2}^{2}\right)-a_{22}\right)-a_{12} a_{21}=0 .
\end{aligned}
$$

The dispersion relation, i.e., the solution of (4.4) with the largest $\operatorname{Re}\left(\sigma_{\mathbf{k}}\right) \geq 0$ is given by

$$
\sigma_{\mathbf{k}}=\frac{1}{2}\left(a_{11}+a_{22}-\left(D_{w}+D_{b}\right)\left(k_{1}^{2}+k_{2}^{2}\right)+i k_{1} v \pm \sqrt{A+i B}\right)
$$

where

$$
\begin{aligned}
A & =\left(a_{11}+a_{22}-\left(D_{w}+D_{b}\right)\left(k_{1}^{2}+k_{2}^{2}\right)\right)^{2}-k_{1}^{2} v^{2} \\
& -4\left(\left(D_{w}\left(k_{1}^{2}+k_{2}^{2}\right)-a_{11}\right)\left(D_{b}\left(k_{1}^{2}+k_{2}^{2}\right)-a_{22}\right)-a_{12} a_{21}\right), \\
B & =2 k_{1} v\left(a_{11}-a_{22}+\left(D_{b}-D_{w}\right)\left(k_{1}^{2}+k_{2}^{2}\right)\right) .
\end{aligned}
$$


Separating the real part and the imaginary part of $\sigma_{\mathbf{k}}$, we obtain

$$
\begin{aligned}
& \operatorname{Re}\left(\sigma_{\mathbf{k}}\right)=\frac{1}{2}\left[a_{11}+a_{22}-\left(D_{w}+D_{b}\right)\left(k_{1}^{2}+k_{2}^{2}\right)+j\left(\frac{\sqrt{A^{2}+B^{2}}+A}{2}\right)^{\frac{1}{2}}\right], \\
& \operatorname{Im}\left(\sigma_{\mathbf{k}}\right)=\frac{1}{2}\left[k_{1} v+j \operatorname{sgn}(B)\left(\frac{\sqrt{A^{2}+B^{2}}-A}{2}\right)^{\frac{1}{2}}\right],
\end{aligned}
$$

where $j= \pm 1$. Then the real part of the eigenvalue with maximum real part is

$$
\operatorname{Re}\left(\sigma_{\mathbf{k}}\right)=\frac{1}{2}\left[a_{11}+a_{22}-\left(D_{w}+D_{b}\right)\left(k_{1}^{2}+k_{2}^{2}\right)+\left(\frac{\sqrt{A^{2}+B^{2}}+A}{2}\right)^{\frac{1}{2}}\right] .
$$

Assume that the stationary homogeneous solution $\left(w^{+}, b^{+}\right)$is linearly stable, i.e.,

$$
\begin{aligned}
\operatorname{Tr}_{\mathbf{k}} & =a_{11}+a_{22}-\left(D_{w}+D_{b}\right)\left(k_{1}^{2}+k_{2}^{2}\right)<0 \\
\operatorname{Det}_{\mathbf{k}} & =D_{w} D_{b}\left(k_{1}^{2}+k_{2}^{2}\right)^{4}-\left(D_{b} a_{11}+D_{w} a_{22}\right)\left(k_{1}^{2}+k_{2}^{2}\right)+a_{11} a_{22}-a_{12} a_{21}>0
\end{aligned}
$$

Based upon these analytical results, we show below that $v$ could induce pattern formation by numerical computations. Choose parameters as $R=14, \lambda=0.2, \mu_{0}=5, \mu_{1}=$ $5.2, D_{w}=1, D_{b}=0.02, k_{1}=3, k_{2}=0$. Then $\operatorname{Re}\left(\sigma_{\mathbf{k}}\right)>0$ if $v$ exceeds the critical value $v_{c}=3.7443$. This means that pattern formation occurs if $v>v_{c}$. Fig. 8 demonstrates that a periodic pattern solution emerges where $v=5.68$.

\section{Conclusion}

In this paper, we study a modified Shnerb's model which incorporates a cross-diffusion term and a monotonously decreasing vegetation death rate. Based on the view of Turing pattern, we discuss the conditions of the diffusion-induced instability and the cross-diffusion-induced instability. Compared with the results of [35], we find that the modified model may undergo much more rich dynamics. Firstly, we study the effects of the diffusion coefficients $D_{w}$ and $D_{b}$ and obtain the diffusion-induced instability region on the $D_{w}-D_{b}$ plane. We find that if the ratio $r=D_{b} / D_{w}$ is large, then as $R$ decreases or $\mu_{1}$ increases there exists a catastrophic shift from vegetation state to bare soil state, whereas for a medium $r$ there are multiple transitions among the vegetation, pattern formation and the bare soil state. We find also that the bare soil state is stable if the rain-fall rate $R$ is small or the "infiltration feedback" parameter $\mu_{1}$ is large which 


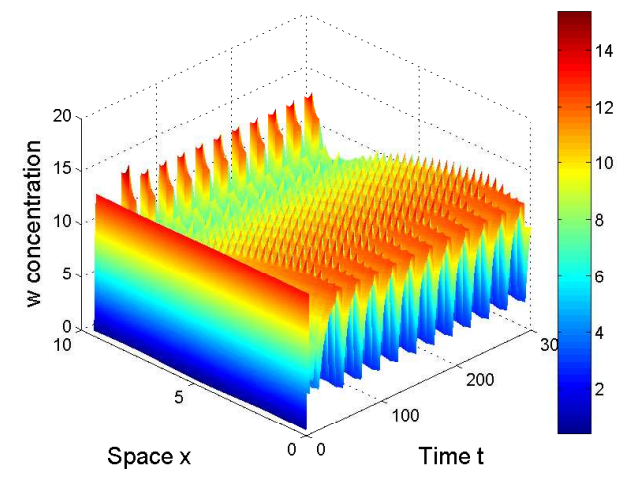

(a)

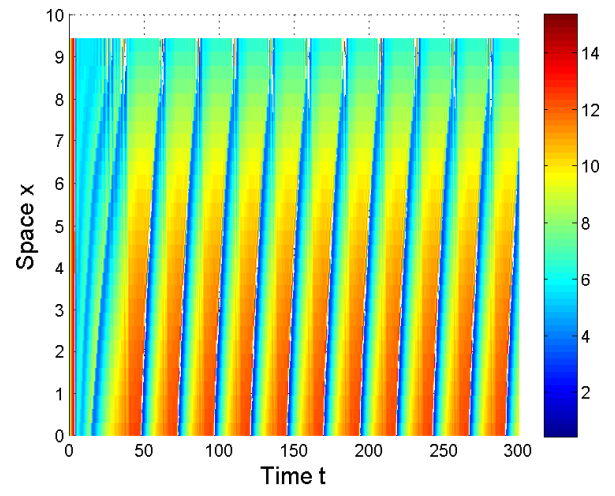

(c)

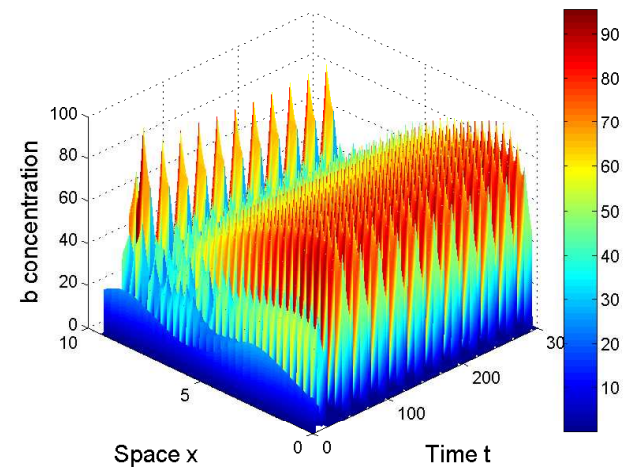

(b)

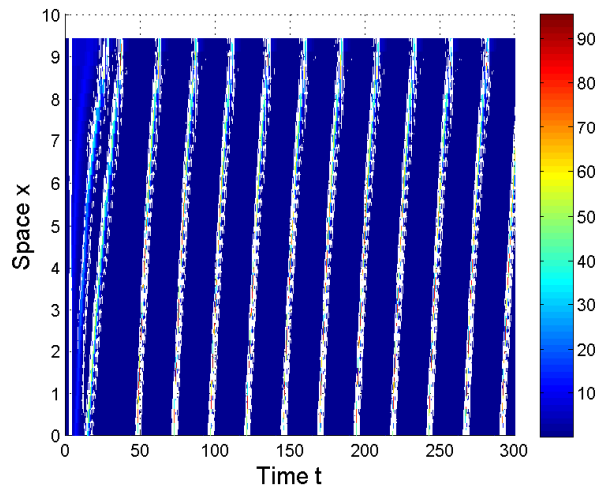

(d)

Figure 8: Pattern solutions of model (4.1). Panel (a) and (b) show a 3D view of the concentration of biomass $w$ and $b$, respectively. Panel (c) and (d) show a 2D view of the concentration of biomass $w$ and $b$, respectively. Parameters: $R=14, \lambda=0.2, D_{w}=$ $1, D_{b}=0.02, \mu_{0}=5, \mu_{1}=5.2, v=5.68$. 
can explain the desertification in arid areas and sandy soil areas. All of the findings indicate that the diffusion may drive the vegetation pattern formation, whereas both the rain-fall rate and the "infiltration feedback" between the water and the biomass may affect the final state of evolution of the vegetation.

Secondly, we illustrate the cross-diffusion-induced instability region on the $\beta-\sqrt{r}$ plane. We find that the range of parameter $r$ for pattern formation is larger for a higher level of $\beta$, where parameter $\beta$ represents the ability of absorption of water due to the roots and captures the "root-augmentation feedback" [25]. The larger $\beta$ means the stronger root-augmentation feedback and the stronger suction ability of roots. This indicates that a vegetation cover comprised of biomass with stronger suction ability is more likely to exhibit pattern formation than a vegetation cover comprised of biomass with weaker suction ability, which is similar to the conclusion of [12].

Finally, we find that the "terrain slope" can also induce the instability of a uniform steady state if the downhill water flow speed $v$ is appropriately large. In this case, the vegetation pattern seems to be periodic. This means that a stripe pattern is formed on hillsides which are consistent with the conclusion of [16].

For a specific shrub system, the nondimensionalized parameter values have been estimated in $[20,35]$. For these parameters, the dynamics of the corresponding kinetic model of (1.3) are simple. Actually, we can show that, for the corresponding kinetic model, the bare-soil equilibrium is globally stable if rain-fall rates are less than the maximum death rate of the biomass, and the positive equilibrium is globally stable if the rain-fall rate is larger than the maximum death rate of the biomass. This means that for the given parameters in $[20,35]$, the biomass is persistent for the corresponding kinetic system of (1.3). However, for system (1.3) the positive equilibrium may lose

stability under the influences of diffusion, cross-diffusion and the "terrain slope" to produce spatiotemporal pattern formation and the diversity of dryland vegetation.

\section{Acknowledgements}

The authors thank Junping Shi for some helpful comments on this work. The authors also thank all the members of College of William and Mary for their supports during visiting. The authors also wish to express their grateful thanks to the anonymous 
referees for their careful reading and some valuable comments and suggestions which greatly improved this work.

\section{References}

[1] Adams, B, Carr, J. Spatial pattern formation in a model of vegetation-climate feedback. Nonlinearity 2003; 16 (4): 1339.

[2] Aguiar MR, Sala OE. Patch structure, dynamics and implications for the functioning of arid ecosystems. Trends Ecol Evol 1999; 14 (7): 273-277.

[3] Barbier, N, Couteron, P, Lejoly, J, Deblauwe, V, Lejeune, O. Self-organized vegetation patterning as a fingerprint of climate and human impact on semiarid ecosystems. J Ecol 2006, 94 (3): 537-547.

[4] Cartení, F, Marasco, A, Bonanomi, G, Mazzoleni, S, Rietkerk, M, Giannino, F. Negative plant soil feedback explaining ring formation in clonal plants. J Theor Biol 2012; 313: 153-161.

[5] Cross, MC, Hohenberg, PC. Pattern formation outside of equilibrium. Rev. Mod Phys 1993; 65 (3): 851.

[6] Deblauwe, V, Barbier, N, Couteron, P, Lejeune, O, Bogaert, J. The global biogeography of semi-arid periodic vegetation patterns. Global Ecol Biogeogr 2008; 17 (6): $715-723$.

[7] Dhooge A, Govaerts, W, Kuznetsov Yu A. MATCONT: A MATLAB package for numerical bifurcation analysis of ODEs. ACM Trans Math Software 2003; 29: 141164.

[8] D'Odorico, P, Laio, F, Ridolfi, L. Noise-induced stability in dryland plant ecosystems. Proc Natl Acad Sci USA 2005; 102 (31): 10819-10822.

[9] Gilad, E, Shachak, M, Meron, E. Dynamics and spatial organization of plant communities in water-limited systems. Theor Popul Biol 2007; 72: 214-230. 
[10] Gilad, E, Von Hardenberg, J, Provenzale, A, Shachak, M, Meron, E. Ecosystem engineers: from pattern formation to habitat creation. Phys Rev Lett 2004; 93: 098105.

[11] Hillel, D. Environmental soil physics. San Diego, California: Academic Press; 1998.

[12] HillerisLambers, R, Rietkerk, MG, Bosch, F van den, Prins, HHT, Kroon, H de. Vegetation pattern formation in semi-arid grazing systems. Ecology 2001, 82: 50-61.

[13] Isham, V, Cox, DR, Rodriguez-Iturbe, I, Porporato, A, Manfreda, S. Representation of space-time variability of soil moisture. Proc Natl Acad Sci USA 2005; 461 (2064): 4035-4055.

[14] Jones, CG, Lawton, JH, Shachak, M. Organisms as ecosystem engineers. Ecosystem Management, 1996; 130-147.

[15] Jones, CG, Lawton, JH, Shachak, M. Positive and negative effects of organisms as physical ecosystem engineers. Ecology 1997; 78: 1946-1957.

[16] Klausmeier, CA. Regular and irregular patterns in semiarid vegetation. Science 1999; 284: 1826-1828.

[17] Koch, AJ, Meinhardt, H. Biological pattern formation: from basic mechanisms to complex structures. Rev Mod Phys 1994; 66: 1481.

[18] Lefever, R, Lejeune, O. On the origin of tiger bush. Bull Math Biol 1997; 59: 263-294.

[19] Liu, Q, Jin, Z, Li, B. Numerical investigation of spatial pattern in a vegetation model with feedback function. J Theoret Biol 2008; 254: 350-360.

[20] Manor, A, Shnerb, NM. Dynamical failure of Turing patterns. Europhys Lett 2006; 74: 837-843.

[21] Marasco, A, Iuorio, A, Cartení, F, Bonanomi, G, Giannino, F, Mazzoleni, S. Water limitation and negative plant-soil feedback explain vegetation patterns along rainfall gradient. Procedia Environ Sci 2013; 19: 139-147. 
[22] Marasco, A, Iuorio, A, Cartení, F, Bonanomi, G, Tartakovsky, DM, Mazzoleni, S, Giannino, F. Vegetation pattern formation due to interactions between water availability and toxicity in plant-soil feedback. Bull Math Biol 2014; 76 (11): 28662883.

[23] Meinhardt, H. Pattern formation in biology: a comparison of models and experiments. Rep Prog Phys 1992; 55: 797.

[24] Meron, E, Gilad, E, von Hardenberg, J, Shachak, M, Zarmi, Y. Vegetation patterns along a rainfall gradient. Chaos, Solitons Fract 2004; 19: 367-376.

[25] Meron, E, Yizhaq, H, Gilad, E. Localized structures in dryland vegetation: forms and functions. Chaos 2007; 17: 037109.

[26] Murray, JD. Mathematical Biology; Biomathematics 19; Second edition; SpringerVerlag; Berlin; 1993.

[27] Murray, JD. Mathematical Biology. II; Interdisciplinary Applied Mathematics 18; Third edition; Springer-Verlag; New York; 2003.

[28] Peters, DPC, Havstad, KM. Nonlinear dynamics in arid and semi-arid systems: interactions among drivers and processes across scales. J Arid Environ 2006; 65 (2): $196-206$.

[29] Rietkerk, M, Van de Koppel, J. Alternate stable states and threshold effects in semi-arid grazing systems. Oikos 1997, 69-76.

[30] Rietkerk, M, Dekker, SC, De Ruiter, PC, van de Koppel, J. Self-organized patchiness and catastrophic shifts in ecosystems. Science 2004; 305: 1926-1929.

[31] Rietkerk, M, Van de Koppel, J. Regular pattern formation in real ecosystems. Trends Ecol Evol 2008; 23 (3): 169-175.

[32] Rodriguez-Iturbe, I, Porporato, A, Ridolfi, L, Isham, V, Coxi, DR. Probabilistic modelling of water balance at a point: the role of climate, soil and vegetation. Proc R Soc Lond Ser A Math Phys Eng Sci 1999; 455 (1990): 3789-3805. 
[33] Scheffer, M, Holmgren, M, Brovkin, V, Claussen, M. Synergy between small-and large-scale feedbacks of vegetation on the water cycle. Global Change Biol 2005, 11 (7): 1003-1012.

[34] Shi, J, Xie, Z, Little, K. Cross-diffusion induced instability and stability in reaction-diffusion systems. J Appl Anal Comput 2011; 1: 95-119.

[35] Shnerb, N, Sarah, P, Lavee, H, Solomon, S. Reactive Glass and Vegetation Patterns. Phys Rev Lett 2003; 90: 038101.

[36] Sun, G, Li, L, Zhang, Z. Spatial dynamics of a vegetation model in an arid flat environment. Nonlinear Dynamics 2013; 73: 2207-2219.

[37] Turing, AM. The chemical basis of morphogenesis. Philos Trans R Soc Lond B Biol Sci 1952; 237: 37-72.

[38] Ursino, N. The influence of soil properties on the formation of unstable vegetation patterns on hillsides of semiarid catchments. Adv Water Resour 2005; 28 (9): 956963.

[39] Von Hardenberg, J, Meron, E, Shachak, M, Zarmi, Y. Diversity of vegetation patterns and desertification. Phys Rev Lett 2001; 87: 198101.

[40] Watt, AS. Pattern and process in the plant community. J Ecol 1947; 1-22.

[41] Yi, F, Wei, J, Shi, J. Bifurcation and spatiotemporal patterns in a homogeneous diffusive predator-prey system. J Differential Equations 2009; 246: 1944-1977. 